\title{
A Mosaic Method on Images Small of Unmanned Aerial Vehicle
}

\author{
Xiang Gao \\ School of Navigation, Information Engineering University, Henan 450001, China. \\ gxyunzui@163.com
}

Keywords: Unmanned aerial images, position and posture information, image registration, transformation matrices, matrix singular value decomposition.

\begin{abstract}
Traditional mosaic methods of unmanned aerial vehicle (UAV) need ground control points, which are difficult to implement in the emergency situation. A new image mosaic method on small unmanned aerial vehicle is proposed. The method uses the Harris algorithm to extract the feature points and refines them by the Best-Bin-First (BBF) algorithm and the random sample consensus (RANSAC) algorithm. The transformation matrices from one image to another are computed by the feature points. The position and posture information of UAV is obtained by the transformation matrices according to the matrix singular value decomposition. The UAV images can be merged by the information. Experimental results demonstrate that the method has advantages in mosaic speed and mosaic quality, and is applied potentially in many areas.
\end{abstract}

\section{Introduction}

The UAV remote sensing system has many advantages, such as flexibility, high image resolution, low cost, etc. It also plays an important role in resource exploration, forest fire, disaster warming, and so on [1]. Because of light weight, the small UAVs are easily affected by air flow and other factors. So the precision of the position and posture is very low. The low precision also increases the calculation quantity of the image mosaic processing and causes low quality of the images mosaic. How to improve the quality of the image mosaic and the speed of the image mosaic processing has become a very tricky problem.

The traditional methods of UVA image mosaic need to set some ground control points. Firstly, the ground control points are found in the UVA images. Then Ortho-Images are obtained by geometric correction. Finally, the image mosaic is completed based on the Ortho-Images. At disaster regions and other typical areas, the ground control points are not easy to be laid. The articles [2, 3] use the flight parameters of UAV to merge and improve the speed of image mosaic. Li Yanshan proposes that the key images are rapidly extracted by using characteristic points [4]. Deng Tao achieves image mosaic by the Harris characteristic point extraction algorithm and the SIFT feature vector calculation method [5]. All of these have improvement in the mosaic speed. But these also are limitations. The image mosaic based on above methods requires a large amount of calculation. And the limitation of carrying equipment directly affect the precision of flight parameters. The low precision leads to larger errors in the processing of images mosaic. Considering above problems, a new mosaic method on small UAV images is proposed. The method extracts the characteristic points of images by the Harris algorithm. The transformation matrices from one image to another are computed according to the points. The position and posture information of the UAV is calculated by the matrices. The original images are corrected to complete the mosaic work by the position and posture information.

\section{Feature Registration}

\subsection{Feature Extraction}

The Harris algorithm uses a small window to extract the characteristic points. By moving the window on the image, it gets rules of gray variation. According to the different rules of the flat, edge and corner, characteristic points are extracted. The algorithm has the advantages in low complexity, high stability, and is less affected by image distortion and rotation [6]. This paper 
chooses the Harris algorithm for extracting the characteristic points. The matrix $\mathrm{M}$ is defined as

$$
\mathrm{M}=\mathrm{G} *\left[\begin{array}{cc}
\mathrm{I}_{\mathrm{x}}^{2} & \mathrm{I}_{\mathrm{x}} \mathrm{I}_{\mathrm{y}} \\
\mathrm{I}_{\mathrm{x}} \mathrm{I}_{\mathrm{y}} & \mathrm{I}_{\mathrm{y}}{ }^{2}
\end{array}\right] \rightarrow \mathrm{A}^{-1}\left[\begin{array}{cc}
\lambda_{1} & 0 \\
0 & \lambda_{2}
\end{array}\right] \mathrm{A},
$$

where $I_{x}, I_{y}$ are the integrals of the image $I$ at the $x, y$ direction, $G$ is the Gauss template, * is convolution between the Gauss model and the function, $\mathrm{A}$ is the rotation factor, $\lambda_{1}, \lambda_{2}$ are the two eigenvalues of the matrix. The corner response function $\mathrm{R}$ is defined as

$$
\mathrm{R}=\operatorname{det} \mathrm{M}-\alpha \operatorname{tr}^{2}(\mathrm{M}),\left\{\begin{array}{c}
\operatorname{det} \mathrm{M}=\lambda_{1} \lambda_{2}=\mathrm{I}_{\mathrm{x}}^{2} \mathrm{I}_{\mathrm{y}}^{2}-\left(\mathrm{I}_{\mathrm{x}} \mathrm{I}_{\mathrm{y}}\right)^{2} \\
\operatorname{tr}(\mathrm{M})=\lambda_{1}+\lambda_{2}=\mathrm{I}_{\mathrm{x}}^{2}+\mathrm{I}_{\mathrm{y}}^{2}
\end{array},\right.
$$

where $\alpha$ is the empirical value between 0.04 and 0.06 in the general. If the value $\mathrm{R}$ is greater than the threshold value $\mathrm{T}$, the point is considered to be the characteristic point.

Most of the characteristic points are clustered at the rich texture region. This paper divides original images into a certain number of grids and extracts the characteristic points in every grid [7]. The automatic threshold can ensure each grids have a certain number of characteristic points. It not only makes the characteristic points more uniform, but also improves the efficiency of detection.

\subsection{Feature Matching}

In order to reduce the matching errors, the distance ratio between the nearest neighbor point and the next nearest neighbor point is used. If the distance ratio is less than threshold, the points are considered to be matching points. The lowering threshold reduces the number of matching points, but the matching effect is much better. Considering the actual situation, the proposed method needs some accurate characteristic point pairs. So the threshold is set to 0.3. The method refines the points by the BBF algorithm and the RANSAC algorithm. The BBF search algorithm can improve search efficiency [7]. The RANSAC algorithm can improve matching accuracy of characteristic points and get more stable results [8].

\section{Position and Posture Calculation}

\subsection{Model}

Image mosaic needs coordinates of characteristic points at the unified coordinate system. This paper uses affine transformation model, which can be seen as a result via translation transformation, rotation transformation and scaling transformation. Model formula is defined as

$$
\left[\begin{array}{c}
\mathrm{x}_{1} \\
\mathrm{y}_{1} \\
1
\end{array}\right]=\mathrm{A}\left[\begin{array}{c}
\mathrm{x}_{2} \\
\mathrm{y}_{2} \\
1
\end{array}\right]=\left[\begin{array}{ccc}
\mathrm{m}_{1} & \mathrm{~m}_{2} & \mathrm{~m}_{3} \\
\mathrm{~m}_{3} & \mathrm{~m}_{4} & \mathrm{~m}_{5} \\
0 & 0 & 1
\end{array}\right]\left[\begin{array}{c}
\mathrm{x}_{2} \\
\mathrm{y}_{2} \\
1
\end{array}\right] \text {. }
$$

Two equations are listed from each pair of points. Six parameters of the model need least three pairs. The model is calculated by 3 pairs of points which are selected randomly. Whether remaining points are needed by the transformation errors. If the error less than 2 pixels, they are needed. The 6 parameters are calculated by indirect adjustment. The number of pairs is limited to 100 in every images. The experimental result shows that the relatively stable results can be obtained with 80 times maximum iterations.

\subsection{Singular Value Decomposition}

The matrix $A$ is operated by singular value decomposition such as

$\mathrm{A}=\mathrm{U} \Sigma \mathrm{V}^{\mathrm{T}}$.

According to the imaging principle of the camera, there is $\mathrm{A}=\mathrm{R}+\mathrm{tn}^{\mathrm{T}} / \mathrm{d}$, where $\mathrm{R}$, $\mathrm{t}$ are respectively the rotation matrix and the move vector, $d$ is the distance from the center of the camera to the ground plane, $\mathrm{n}$ is unit normal vector of ground plane, $\lambda_{1}, \lambda_{2}, \lambda_{3}$ are the eigenvalues of $\mathrm{A}^{\mathrm{T}} \mathrm{A}$ and they are greater than 0 . Formula (4) can also be expressed as

$$
\Sigma=\mathrm{d}^{\prime} \mathrm{R}^{\prime}+\mathrm{t}^{\prime} \mathrm{n}^{\mathrm{T}} \text {, }
$$

where $\mathrm{R}=\mathrm{sUR} \mathrm{V}^{\mathrm{T}}, \mathrm{t}=\mathrm{Ut} \mathrm{t}^{\prime}, \mathrm{n}=\mathrm{Vn}^{\prime}, \mathrm{d}=\mathrm{sd}^{\prime}, \mathrm{s}=\operatorname{detUdetV}$. If $\mathrm{n}^{\prime}=\mathrm{x}_{1} \mathrm{e}_{1}+\mathrm{x}_{2} \mathrm{e}_{2}+\mathrm{x}_{3} \mathrm{e}_{3}$, there are three equations

$$
\lambda_{i} e_{i}=d^{\prime} R^{\prime} e_{i}+t^{\prime} x_{i}(i=1,2,3) .
$$


For making the equation with nonzero solutions, there are

$\left(d^{2}-\lambda_{1}^{2}\right)\left(d^{2}-\lambda_{2}^{2}\right)\left(d^{2}-\lambda_{3}^{2}\right)=0$.

(1)If $\lambda_{1} \neq \lambda_{2} \neq \lambda_{3}$ and $d^{\prime}= \pm \lambda_{2}$

$$
\begin{aligned}
& \left\{\begin{array} { l } 
{ \mathrm { R } ^ { \prime } = [ \begin{array} { c c c } 
{ \operatorname { c o s } \theta } & { 0 } & { - \operatorname { s i n } \theta } \\
{ 0 } & { 1 } & { 0 } \\
{ \operatorname { s i n } \theta } & { 0 } & { \operatorname { c o s } \theta }
\end{array} ] } \\
{ \mathrm { t } ^ { \prime } = ( \lambda _ { 1 } - \lambda _ { 3 } ) ( \mathrm { x } _ { 1 } , 0 , - \mathrm { x } _ { 3 } ) ^ { \mathrm { T } } }
\end{array} \quad \left\{\begin{array}{l}
\sin \theta=\left(\lambda_{1}-\lambda_{3}\right) \frac{\mathrm{x}_{1} \mathrm{x}_{3}}{\lambda_{2}} \\
\cos \theta=\frac{\lambda_{1} \mathrm{x}_{3}^{2}+\lambda_{3} \mathrm{x}_{1}^{2}}{\lambda_{2}}
\end{array} .\right.\right. \\
& \text { (2)If } \lambda_{1}=\lambda_{2} \neq \lambda_{3} \text { and d' }= \pm \lambda_{2} \quad x_{1}=x_{2}=0, x_{3}= \pm 1 \\
& \left\{\begin{array}{c}
\mathrm{R}^{\prime}=\mathrm{I} \\
\mathrm{t}^{\prime}=\left(\lambda_{3}-\lambda_{1}\right) \mathrm{n}^{\prime}
\end{array}\right.
\end{aligned}
$$

In this way, the rotation matrix $\mathrm{R}$ and the move vector $\mathrm{t}$ can be obtained. Pitch angle, roll angle and yaw angle are also obtained by the rotation matrix. The geometric correction images will be merged to gain a panoramic image.

\section{Experiment and Analysis}

The UAV images $\mathrm{I}_{1} \sim \mathrm{I}_{4}$ are shot at $\mathrm{O}_{1}-\mathrm{O}_{4}$ positions. The fixed flight height is set about to 200 meters. The size of an image is $4608 * 2592$ pixels. The main ground objects are buildings, roads and groves in the survey area. So the survey area is representative. Most of the characteristic points are the ground points. The experimental platform is Intel (R) Core (TM) i7-4720HQ CPU 2.60GHz which has 8GB memory. The experimental software is MATLAB R2010b.

(1) Characteristic points are obtained by the Harris algorithm. Characteristic points of adjacent images are matched and refined based on the BBF search algorithm and the RANSAC algorithm.

(2) According to the space coordinates of characteristic points, the position and posture information of UAV is calculated.

(3) The distortion of the UAV images are corrected by the position and posture information. And the multiple images are merged.

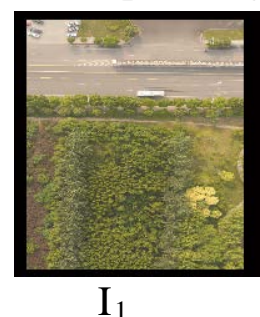

$\mathrm{I}_{1}$

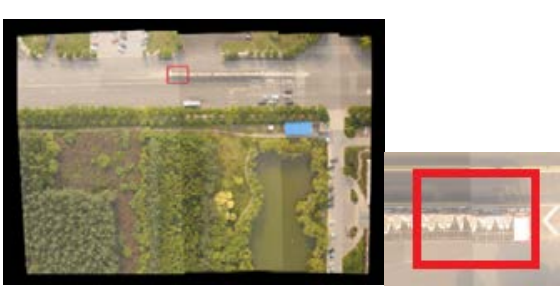

a Local image of this paper
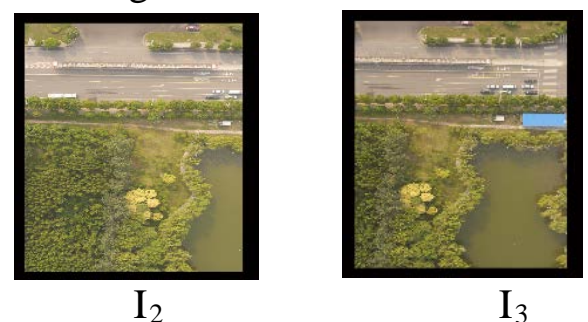

$\mathrm{I}_{3}$

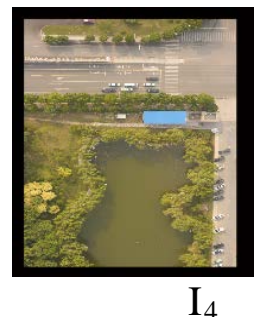

$\mathrm{I}_{4}$

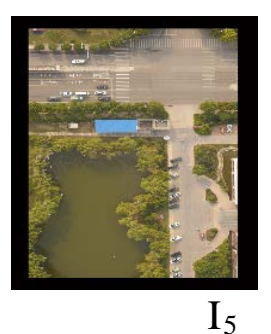

$\mathrm{I}_{5}$

Fig. 1 Original images of UAV

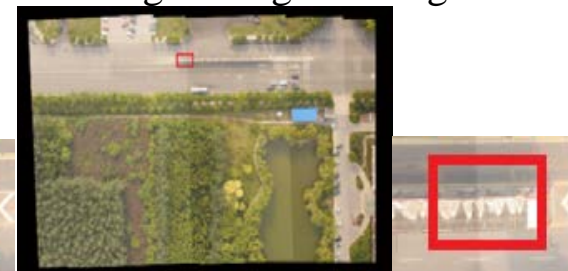

b Local image by SIFT

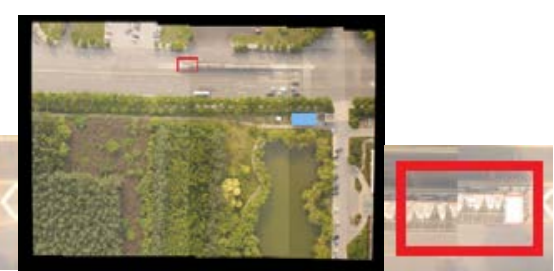

c Local image of direct matching

Fig.2 Detail of the image mosaic

Table 1 Time consumption of three method

\begin{tabular}{cccc}
\hline $\begin{array}{c}\text { The number } \\
\text { of image }\end{array}$ & $\begin{array}{c}\text { The method of this } \\
\text { paper }\end{array}$ & $\begin{array}{c}\text { SIFT feature } \\
\text { matching method }\end{array}$ & $\begin{array}{c}\text { the direct } \\
\text { matching method }\end{array}$ \\
\hline 2 & $3.08 \mathrm{~min}$ & $5.12 \mathrm{~min}$ & $1.12 \mathrm{~min}$ \\
3 & $4.94 \mathrm{~min}$ & $8.86 \mathrm{~min}$ & $2.09 \mathrm{~min}$ \\
4 & $7.39 \mathrm{~min}$ & $13.27 \mathrm{~min}$ & $3.43 \mathrm{~min}$ \\
5 & $10.46 \mathrm{~min}$ & $18.65 \mathrm{~min}$ & $4.29 \mathrm{~min}$ \\
\hline
\end{tabular}


The proposed method is compared with the SIFT feature matching method and the direct matching method. The method has almost the same mosaic effect as the SIFT method. But there are some mosaic errors in the details (such as bus station in Figure 2). Which mainly are the error of UAV flight parameters calculation. However the time consumption table shows that the proposed method takes less time than the SIFT method. Compared with the direct mosaic effect, the proposed method has been greatly improved in the mosaic quality.

\section{Summary}

This paper proposes a mosaic method on small unmanned aerial vehicle images. The experiment shows that the method can effectively enhance the mosaic effect. The low complex algorithm and the thought of grid are used in the feature extraction, which ensure certainly the image mosaic efficiency. The matrices are calculated by the characteristic points. But if the posture change of reference image is too large, the correction value of UAV images is difficult to calculate. Further research is needed on how to choose a suitable reference image.

\section{References}

[1]. ZHOU Jieping, GONG Jianhua, WANG Tao, et al. Remote Sensing Image Acquiring and Visualization Management System for the Area Affected by $5^{\circ} 12$ Wenchuan Earthquake. Journal of Remote Sensing. Vol. 12 (2008) No. 6, p.877-884.

[2]. HE Bei, WANG Guijin, SHEN Yongling, et al. Real-Time Mosaicing for Aerial Videos Combined with Geographical Parameters. Journal of Applied Sciences. Vol. 30 (2012) No. 2, p.151-157.

[3]. ZHANG Yan, LI Jianzeng, LI Deliang, et al. Research on UAV Targeting Based on POS and Image Matching[J]. Journal of Ordnance Engineering College. Vol. 27 (2015) No. 1, p.39-45.

[4]. LI Yanshan, PEI Jihong, XIE Weixin, et al. A New Fast Automatic Mosaic Method on Unmanned Aerial Vehicle Images. Acta Electronica Sinica. Vol. 40 (2012) No. 5, p.935-940.

[5]. DENG Tao, HOU Dongping, LE Qinlan, et al. Automatic Mosaic of Small Unmanned Aerial Vehicle Images Based on SIFT and Harris Algorithm [J]. Journal of Information Engineering University. Vol. 16 (2015) No. 3, p.321-326.

[6]. YAO Guobiao, YANG Huachao, ZHANG Lei. Wide baseline image sequence least squares matching algorithm of Harris- Laplace. Science of Surveying and Mapping. Vol. 36 (2011) No. 6, p.141-143.

[7]. Beis J S, Lowe D G. Shape indexing using approximate nearest-neighbour search in high-dimensional spaces. Conference on Computer Vision \& Pattern Recognition. San Juan, 1997, p. 1000-1006.

[8]. Fiscler M A, Bolles R C. Random sample consensus: A Paradigm for model fitting with apphcatlons to image analysis and automated cartography. Graphics and Image Processing. Vol. 24 (1981) No. 6, p.381-395.

[9]. Benhimane S, Malis E. Homography-based 2D visual servoing. Proceedings 2006 IEEE International Conference on Robotics and Automation. Orlando, 2006, p. 2397-2402. 